\title{
New data on sulphosalts from hydrothermal siderite-type veins in the Spišsko-gemerské rudohorie Mts. (eastern Slovakia): 1. Nuffieldite and aikinite from Slovinky-Došt’avná vein
}

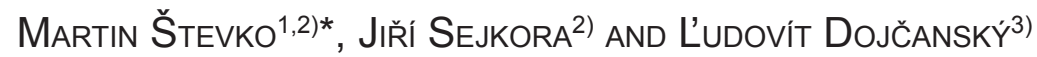 \\ 1)Earth Science Institute, Slovak Academy of Sciences, Dúbravská cesta 9, 84005 Bratislava, Slovak Republic; \\ *e-mail: martin.stevko@savba.sk \\ 2)Department of Mineralogy and Petrology, National Museum, Cirkusová 1740, 19300 Praha 9 - Horní Počernice, \\ Czech Republic \\ 3)Strojnícka 1, 05201 Spišská Nová Ves, Slovak Republic
}

ŠTeVko M, SEJKora J, DoJČAnSkÝ L' (2021) New data on sulphosalts from hydrothermal siderite-type veins in the Spišsko-gemerské rudohorie Mts. (eastern Slovakia): 1. Nuffieldite and aikinite from Slovinky-Došt’avná vein. Bull Mineral Petrolog 29(1): 108-114 ISSN 2570-7337

\begin{abstract}
A new occurrence of nuffieldite was recently discovered in a siderite-type hydrothermal vein with sulphides in Došt'avná near Slovinky, Spišsko-gemerské rudohorie Mts., Spišská Nová Ves Co., Košice Region, Slovakia. It forms lead-gray acicular crystals reaching up to $1 \mathrm{~cm}$ in size or aggregates up to $2.5 \mathrm{~cm}$, which are enclosed in quartz-siderite matrix together with chalcopyrite, pyrite, tourmaline and chlorite. Nuffieldite is frequently replaced by minor aikinite, galena and native bismuth. The refined unit-cell parameters of nuffieldite (for the orthorhombic space group $P b n m$ ) are: a 14.5313(16) $\AA, b$ 21.454(2) $\AA, c$ 4.0500(6) $\AA$ and $V$ 1262.58(19) $\AA^{3}$. The average ( $n=145$ analyses) empirical formula of nuffieldite from Slovinky-Došt'avná based on $\mathrm{Pb}+\mathrm{Bi}+\mathrm{Sb}=5$ apfu is corresponding to $\mathrm{Cu}_{1.30} \mathrm{~Pb}_{2.00} \mathrm{Bi}_{2.00}\left(\mathrm{~Pb}_{0.30} \mathrm{Bi}_{0.30}\right.$ $\left.\mathrm{Sb}_{0.40}\right)_{1.00}\left(\mathrm{~S}_{7.12} \mathrm{Se}_{0.03}\right)_{7.15}$. Aikinite forms microscopic, anhedral to subhedral grains or aggregates replacing nuffieldite. The average $(n=29)$ empirical formula of studied aikinite based on $(\mathrm{Cu}+\mathrm{Pb}) / 2+(\mathrm{Sb}+\mathrm{Bi})=8$ apfu is $\mathrm{Pb}_{3.83} \mathrm{Cu}_{3.76}\left(\mathrm{Bi}_{4.12}\right.$ $\left.\mathrm{Sb}_{0.09}\right)_{4.21}\left(\mathrm{~S}_{12.40} \mathrm{Se}_{0.03}\right)_{12.03}$
\end{abstract}

Key words: nuffieldite, aikinite, sulphosalts, X-ray powder data, chemical composition, Došt'avná vein, Slovinky, Spišsko-gemerské rudohorie Mts., Slovak Republic

Received 4. 2. 2021; accepted 27. 5. 2021

\section{Introduction}

The Spišsko-gemerské rudohorie Mts. represent one of the most important accumulations of ore deposits in whole Carpathian mountain range. There are more than 1200 hydrothermal ore veins known within this relatively small area, with two major types of mineralization: siderite -type carbonate-quartz veins with sulphides (extensively exploited in Dobšiná, Štítnik, Rákoš, Rožňava, Drnava, Rudňany, Novoveská Huta, Hnilčík, Henclová, Prakovce, Gelnica, Slovinky, Medzev etc.) and quartz-stibnite veins (Betliar, Čučma, Štofova dolina, Helcmanovce, Poproč or Zlatá Idka). Furthermore, Sn-Mo-W bearing greisens or hydrothermal veins, hydrothermal veins with U-REE mineralization as well as strata-bound VMS pyrite-Cu-Pb-Zn ore mineralization and hydrothermal-metasomatic bodies of siderite and magnesite \pm talc are present (Varček 1962; Chovan et al. 1994; Grecula et al. 1995; Rojkovič 1997).

All of the above mentioned types of ore mineralization contain various sulphosalts mostly as accessory ore minerals. Abundant presence of minerals of tetrahedritetennantite series (especially $\mathrm{Fe}, \mathrm{Zn}$ and locally also $\mathrm{Hg}$ dominant members) is very typical feature of the siderite -type veins (e.g. Bernard 1958, 1961; Varček 1957, 1959, 1960; Novák 1959, 1967; Trdlička 1967; Háber 1980; Cambel et al. 1985; Peterec 1990; Miškovic 1991; Háber et al. 1993; Grecula et al. 1995; Antal 2002a, b; Pršek
2008; Pršek, Biroň 2007; Pršek, Lauko 2009; Števko et al. 2015; Mikuš et al. 2018; Števko, Sejkora 2020). Bi sulphosalts are also quite common, especially minerals of bismuthinite-aikinite series (e.g. Paděra et al. 1955; Kupčík et al. 1969; Hurný, Krištín 1978; Mumme, Žák 1983; Antal 1991; Beňka, Siman 1994; Pršek 2008; Števko et al. 2015; Mikuš et al. 2018, 2019) and kobellite homologous series (e.g. Trdlička, Kupka 1957; Hak, Kupka 1958; Novák 1961; Trdlička et al. 1962; Václav 1964; Zábranský, Radzo 1966; Háber, Streško 1969; Háber 1980; Jeleň 1991; Pršek 2008; Pršek, Peterec 2008; Mikuš et al. 2018, 2019). Other Bi sulphosalts like cosalite (Bernard 1964, Háber 1980), galenobismutite (Antal 1991, Pršek 2008), jaskólskiite (Pršek, Biroň 2007), nuffieldite (Pršek et al. 2006) or wittichenite (Háber 1978; Kozub et al. 2011) are rare. Chalcostibite is infrequent too (Sejkora et al. 2011; Mikuš et al. 2018). Unusual assemblage of $\mathrm{Ag}-\mathrm{Bi}$ sulphosalts (matildite, gustavite and benjaminite) was recently described from Kobaltová vein near Medzev by Mikuš et al. (2019). The most common Pb sulphosalts at the siderite-type veins are bournonite, jamesonite and boulangerite (e.g. Zimányi 1914; Novák 1962; Trdlička 1967; Kupčík et al. 1969; Háber 1980; Miškovic 1990; Pršek, Biroň 2007; Pršek, Peterec 2008; Sejkora et al. 2011; Mikuš et al. 2018, 2019), whereas berthierite and garavellite (Mikuš et al. 2018), meneghinite (Beňka, Si- 
man 1994) or zinkenite and scainiite (Sejkora et al. 2011) are scarce. Rare $\mathrm{Hg}$ sulphosalts, marruccite (Sejkora et al. 2011) and grumiplucite (Števko et al. 2015) were also recently identified.

In this series of papers, we would like to present new data on various sulphosalts from newly discovered localities in the Spišsko-gemerské rudohorie Mts.

\section{Geological setting}

The Došt'avná vein is located in the Došt'avná valley, around $7 \mathrm{~km} \mathrm{SW}$ of the Slovinky village and $1 \mathrm{~km} \mathrm{NW}$ of the Orlí vrch hill (1043 m a.s.I.) in the Spišsko-gemerské rudohorie Mts., Spišská Nová Ves Co., Košice Region, Slovakia. Samples of ore mineralization with sulphosalts were collected at the dump of abandoned adit (Fig. 1) located in the eastern part of the Došt'avná vein/vein system. GPS coordinates (WGS84) of this dump are: $48.843134^{\circ} \mathrm{N}$ a $20.768606^{\circ} \mathrm{E}, 741 \mathrm{~m}$ asl.

The Došt'avná vein is one of the minor siderite-type hydrothermal carbonate-quartz ore veins with sulphides, explored for $\mathrm{Cu}$ ores mainly in $19^{\text {th }}$ century. According to Grecula et al. (1995) it is belonging to the Zlatá vein zone. Based on our recent detailed mapping of old mining works it is clear that rather than one vein, a system of se- veral subparallel hydrothermal veins is developed in the Doštavná valley area. The E-W to NE-SW trending hydrothermal veins are mostly up to $0.6 \mathrm{~m}$ thick and are hosted by Early Paleozoic rocks of the Gelnica group, predominantly metarhyolite tuffs and phyllites of the Vlachovo Formation (Hurný 1973, 1974; Bajaník et al. 1984; Grecula et al. 1995). Mineralogy of the Doštavná vein was studied by Hurný $(1973,1974)$ and Hurný, Krištín (1978). The dominant gangue minerals are quartz, siderite and ankerite accompanied by minor amounts of chlorite, tourmaline, dickite and xenotime. Chalcopyrite and pyrite are the most frequent ore minerals associated with minor aikinite, hematite, magnetite, tetrahedrite and microscopic inclusions of native bismuth (Hurný 1973, 1974; Hurný, Krištín1978).

\section{Analytical methods}

The powder X-ray diffraction data of nuffieldite were collected on a Bruker D8 Advance diffractometer (National Museum, Prague, Czech Republic) with a solid-state 1D LynxEye detector using CuKa radiation and operating at $40 \mathrm{kV}$ and $40 \mathrm{~mA}$. The powder patterns were collected using Bragg-Brentano geometry in the range $3-70^{\circ} 2 \theta$, in $0.01^{\circ}$ steps with a counting time of $20 \mathrm{~s}$ per step. Positions

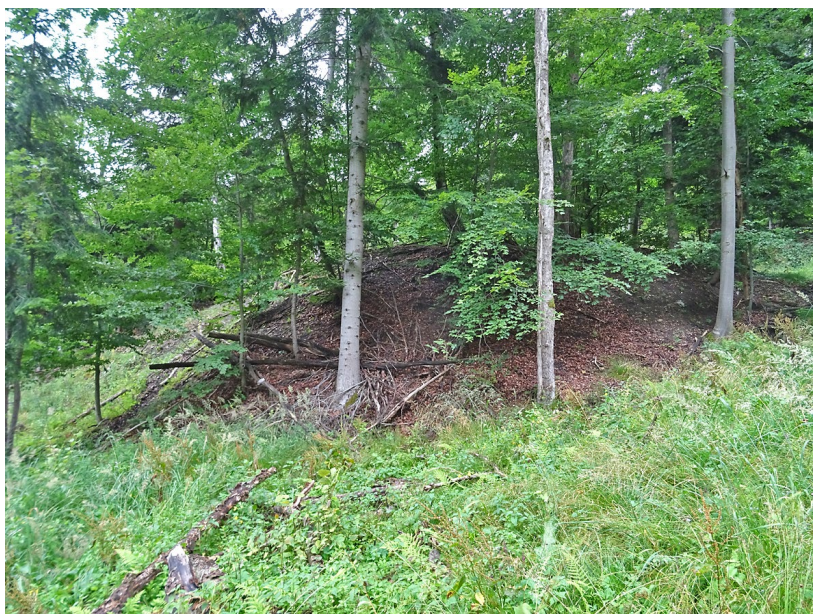

Fig. 1 View on dump where samples with nuffieldite were collected. Photo by M. Števko, August 2020.

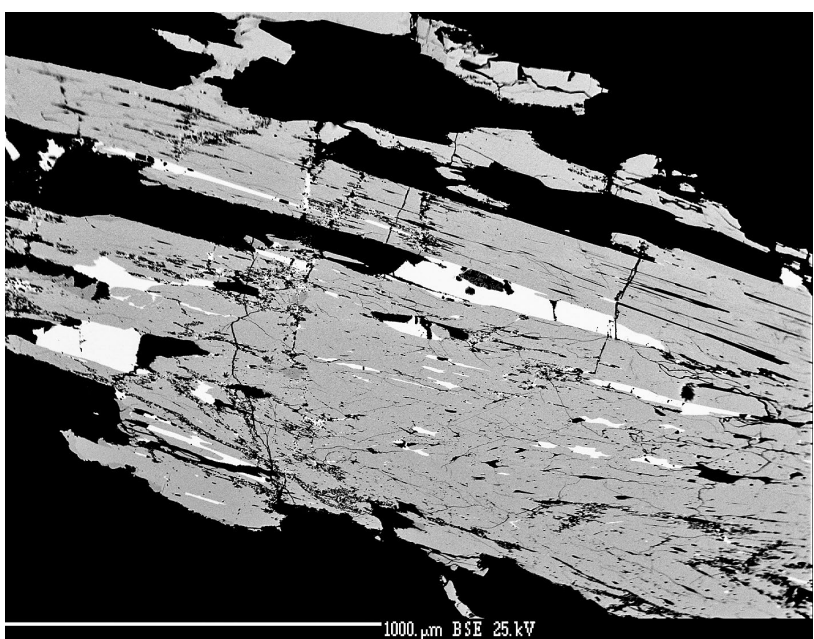

Fig. 3 Homogenous acicular crystals of nuffieldite (light gray) with galena (white) in quartz (black). BSE image by J. Sejkora.

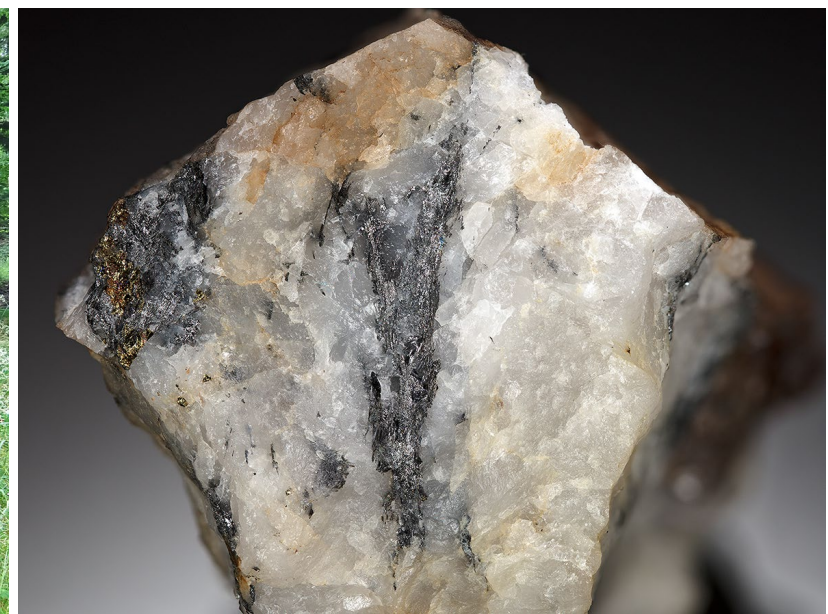

Fig. 2 Aggregate of acicular crystals of nuffieldite enclosed with chalcopyrite in quartz. Nuffieldite aggregate is $2.5 \mathrm{~cm}$ long. Photo by P. Škácha.

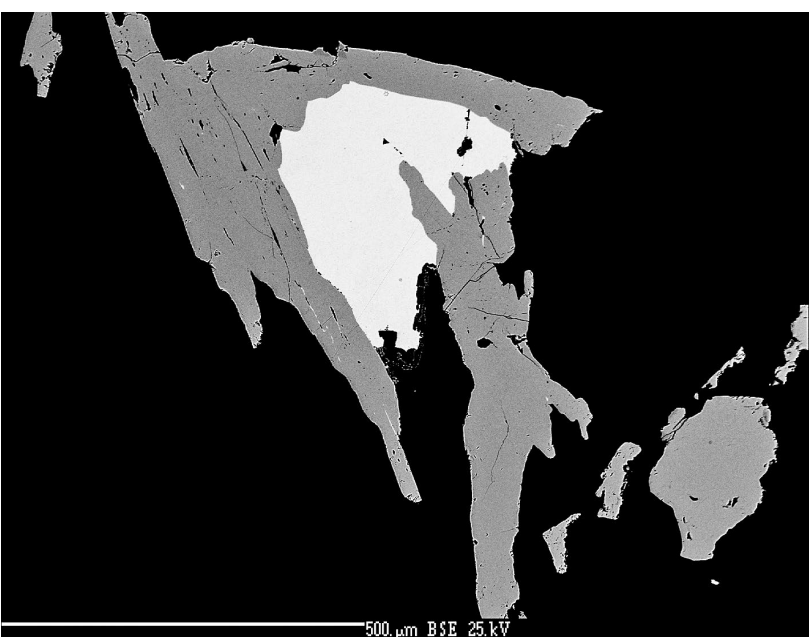

Fig. 4 Aggregates of nuffieldite (gray) with galena (white) in quartz (black). BSE image by J. Sejkora. 
and intensities of reflections were found and refined using the PearsonVII profile-shape function with the ZDS program package (Ondruš 1993) and the unit-cell parameters were refined by the leastsquares algorithm implemented by Burnham (1962). The experimental powder pattern was indexed in line with the calculated values of intensities obtained from the crystal structure of nuffieldite (Moëlo et al. 1997), based on Lazy Pulverix program (Yvon et al. 1977).
The chemical analyses of nuffieldite and aikinite were performed using a Cameca SX100 electron microprobe (National Museum, Prague, Czech Republic) operating in the wave-dispersive (WDS) mode $(25 \mathrm{kV}, 20 \mathrm{nA}$ and 0.7 $\mu \mathrm{m}$ wide beam). The following standards and $\mathrm{X}$-ray lines were used to minimize line overlaps: $\mathrm{Ag}(\mathrm{AgL} \alpha), \mathrm{Bi}_{2} \mathrm{Se}_{3}$ $(\mathrm{Bi} M \beta)$, CdTe $(\mathrm{Cd} L \alpha)$, Co (CoKa), CuFeS $(\mathrm{CuK \alpha}, \mathrm{S} K \alpha)$, $\mathrm{FeS}_{2}(\mathrm{FeK} \alpha), \mathrm{GaAs}(\mathrm{GaL} \alpha)$, Ge $(\mathrm{Ge} L \alpha)$, $\mathrm{HgTe}(\mathrm{Hg} L \alpha)$,

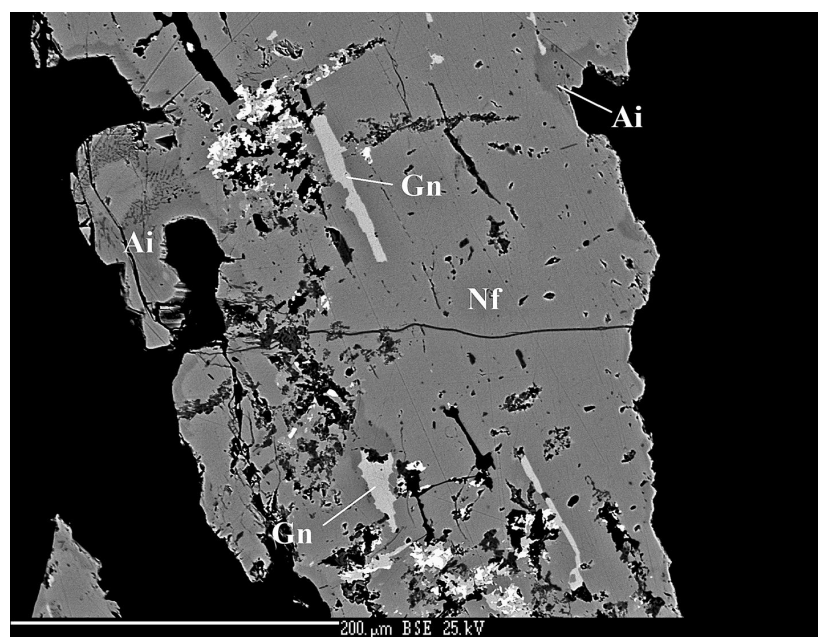

Fig. 5 Nuffieldite (Nf) replaced by aikinite (Ai), galena $(\mathrm{Gn})$ and native bismuth (tiny white inclusions). BSE image by J. Sejkora.

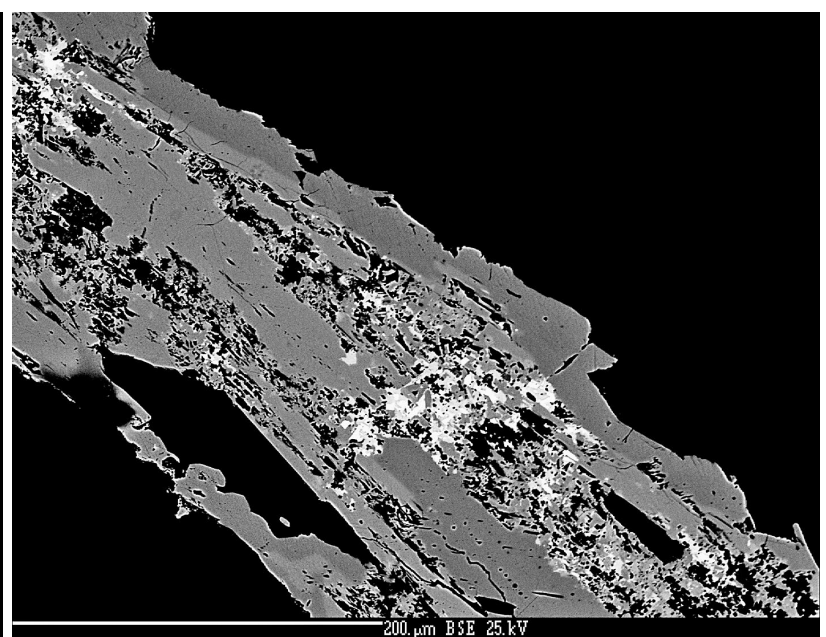

Fig. 6 Nuffieldite (light gray) replaced by aikinite (dark gray) and native bismuth (white). BSE photo by J. Sejkora.

Table $1 X$-ray powder diffraction data of nuffieldite from Slovinky

\begin{tabular}{|c|c|c|c|c|c|c|c|c|c|c|c|c|c|c|c|c|c|}
\hline$I_{\text {obs. }}$ & $d_{o b s .}$ & $d_{\text {calc. }}$ & $h$ & $k$ & I & $I_{o b s .}$ & $d_{o b s .}$ & $d_{\text {calc. }}$ & $h$ & $k$ & 1 & $I_{\text {obs. }}$ & $d_{o b s .}$ & $d_{\text {calc. }}$ & $h$ & $k$ & \\
\hline 9.3 & 7.286 & 7.266 & 2 & 0 & 0 & 7.4 & 2.6373 & 2.6372 & 1 & 8 & 0 & 6.2 & 1.8204 & 1.8206 & 7 & 2 & \\
\hline 0.3 & .904 & 6.882 & 2 & 1 & 0 & 2.1 & 2.5903 & 2.5899 & & 7 & & 3.2 & 1.8101 & .8076 & & & \\
\hline 1.1 & 370 & 5.363 & 0 & 4 & 0 & 33.5 & 2.5488 & 2.5483 & 4 & 6 & 0 & 2.9 & 1.7968 & 1.7970 & 6 & 6 & \\
\hline 2.9 & 5.102 & 5.097 & 2 & 3 & 0 & 9.5 & 2.5299 & 2.5295 & 4 & 3 & 1 & 3.0 & 1.7915 & 1.7909 & 8 & & \\
\hline 2.7 & 4.730 & 4.725 & 3 & 1 & 0 & \multirow{2}{*}{3.3} & \multirow{2}{*}{2.5154} & 2.5165 & & 5 & 1 & 2.7 & 1.7890 & 1.7882 & 4 & $y$ & \\
\hline 9.5 & 4.319 & 4.315 & 2 & 4 & 0 & & & 2.5148 & 2 & 6 & 1 & 0.5 & 1.7707 & 1.7688 & 4 & 0 & \\
\hline 6.6 & 4.119 & 4.115 & 1 & 5 & 0 & 9.6 & 2.4080 & 2.4101 & 1 & 7 & 1 & 6.1 & 1.7615 & 1.7628 & 4 & 1 & \\
\hline 66.1 & 4.014 & 4.010 & 3 & 3 & 0 & \multirow{2}{*}{7.4} & \multirow{2}{*}{2.3629} & 2.3624 & 0 & 2 & 0 & 0.0 & 1.7615 & 1.7620 & 0 & 6 & \\
\hline 2.8 & 3.791 & 3.789 & 0 & 2 & 1 & & & 2.3612 & 5 & 0 & 1 & 5.8 & 1.7465 & 1.7467 & 7 & 4 & \\
\hline 60.8 & 3.696 & 3.695 & 2 & 5 & 0 & 10.0 & 2.3432 & 2.3470 & 5 & 1 & 1 & \multirow{2}{*}{4.4} & \multirow{2}{*}{1.7187} & 1.7184 & 4 & 11 & \\
\hline 24.3 & 3.670 & 666 & 1 & 2 & 1 & 15.5 & 2.3064 & 2.3060 & & 2 & 1 & & & 1.7187 & 7 & 7 & \\
\hline 45.8 & 3.635 & 3.633 & 4 & 0 & 0 & 1.5 & 2.2936 & 2.2939 & & 3 & 0 & 2.4 & 1.7126 & 1.7130 & 3 & 5 & \\
\hline \multirow{2}{*}{100.0} & \multirow{2}{*}{3.580} & 3.582 & 4 & 1 & 0 & 2.8 & 2.2883 & 2.2878 & 4 & 5 & 1 & 3.7 & 1.7075 & 1.7080 & 2 & 11 & \\
\hline & & 3.576 & 0 & 6 & 0 & 9.1 & 2.2359 & 2.2360 & 0 & 8 & 1 & 3.4 & 1.6986 & 1.6989 & 6 & 9 & \\
\hline 10.5 & 3.491 & 3.490 & 2 & 1 & 1 & 7.8 & 2.2095 & 2.2100 & 1 & 8 & 1 & 2.5 & 1.6775 & 1.6775 & 5 & 9 & \\
\hline 7.3 & 3.443 & 3.441 & 4 & 2 & 0 & 5.6 & 2.1818 & 2.1819 & & 7 & 1 & 4.4 & 1.6523 & 1.6524 & 8 & 1 & \\
\hline 14.1 & 3.428 & 3.425 & 1 & 3 & 1 & 5.9 & 2.1600 & 2.1611 & & 4 & 1 & \multirow{2}{*}{6.8} & \multirow{2}{*}{1.6411} & 1.6413 & 7 & 6 & \\
\hline 8.6 & 3.361 & 3.360 & 2 & 2 & 1 & 7.1 & 2.1379 & 2.1371 & 2 & 8 & 1 & & & 1.6397 & 1 & 13 & \\
\hline 16.4 & 3.213 & 3.212 & 3 & 5 & 0 & \multirow{2}{*}{5.3} & \multirow{2}{*}{2.1090} & 2.1089 & 5 & 7 & 0 & 2.8 & 1.6348 & 1.6353 & 4 & 5 & \\
\hline 35.4 & 3.172 & 3.171 & 2 & 3 & 1 & & & 2.1091 & 6 & 5 & 0 & \multirow{2}{*}{1.9} & \multirow{2}{*}{1.6054} & 1.6061 & 1 & 8 & \\
\hline 16.0 & 3.009 & 3.008 & 4 & 4 & 0 & 2.9 & 2.0572 & 2.0575 & 2 & 10 & 0 & & & 1.6059 & 6 & 10 & \\
\hline 8.7 & 3.000 & .999 & 1 & 7 & 0 & 12.7 & 2.0394 & 2.0406 & & 2 & 1 & 2.6 & 1.5848 & 1.5854 & 4 & 6 & \\
\hline 12.0 & 2.985 & 2.984 & 3 & 2 & 1 & 12.5 & 2.0247 & 2.0250 & o & 0 & 2 & \multirow{2}{*}{4.4} & \multirow{2}{*}{1.5459} & 1.5460 & 8 & 5 & \\
\hline 9.9 & 2.954 & 2.953 & 2 & 4 & 1 & \multirow{2}{*}{8.9} & \multirow{2}{*}{1.9938} & 1.9930 & 4 & 9 & 0 & & & 1.5461 & 9 & 4 & \\
\hline 17.4 & 2.886 & 2.887 & 1 & 5 & 1 & & & 1.9936 & 7 & 3 & 0 & \multirow{2}{*}{1.1} & \multirow{2}{*}{1.5321} & 1.5324 & 0 & 14 & \\
\hline 10.6 & 2.850 & 2.850 & 3 & 3 & 1 & 3.5 & 1.9764 & 1.9768 & 2 & 9 & 1 & & & 1.5320 & 4 & 7 & \\
\hline 7.6 & 2.824 & 2.824 & 2 & 7 & 0 & 12.5 & 1.9708 & 1.9709 & 5 & 8 & 0 & 3.4 & & 1.5199 & 1 & 13 & \\
\hline 16.2 & 2.806 & 2.805 & 5 & 2 & 0 & 13.5 & 1.9363 & 1.9360 & 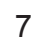 & 4 & 0 & 4.7 & 1.4957 & 1.4961 & 9 & 1 & \\
\hline 35.7 & 2.773 & 2.773 & 4 & 5 & 0 & 9.1 & 1.8909 & 1.8913 & & 9 & 1 & 2.4 & 1.4911 & 1.4918 & 7 & 10 & \\
\hline 22.9 & 2.730 & 2.729 & 2 & 5 & 1 & 26.0 & 1.8833 & 1.8837 & 2 & 11 & 0 & 2.5 & 1.4572 & 1.4575 & 3 & 13 & \\
\hline 8.6 & 2.689 & 2.688 & 3 & 4 & 1 & 10.7 & 1.8703 & 1.8705 & & 7 & 1 & & & & & & \\
\hline 10.6 & 2.681 & 2.682 & 0 & 8 & 0 & 6.1 & 1.8431 & 1.8431 & 5 & 9 & 0 & & & & & & \\
\hline
\end{tabular}


$\ln \mathrm{As}(\operatorname{In} L \alpha), \mathrm{Mn}(\mathrm{MnK} \alpha), \mathrm{NaCl}(\mathrm{Cl} K \alpha), \mathrm{NiAs}(\mathrm{As} L \beta), \mathrm{Ni}$ $(\mathrm{NiK} \alpha), \mathrm{PbS}(\mathrm{PbM} \alpha), \mathrm{PbSe}(\mathrm{Se} L \beta), \mathrm{PbTe}(\mathrm{Te} L \alpha), \mathrm{Sb}_{2} \mathrm{~S}_{3}$ $(\mathrm{Sb} L \alpha)$, Sn $(\mathrm{Sn} L \alpha)$ and $\mathrm{ZnS}(\mathrm{ZnK} \alpha)$. Contents of the above-listed elements, which are not included in the tables, were analysed quantitatively, but their contents were below the detection limit (ca. $0.03-0.05$ wt. \% for individual elements). Raw intensities were converted to the concentrations of elements using automatic "PAP" matrix-correction software (Pouchou, Pichoir 1985). The hypothetical percentage of aikinite end member $n_{\text {aik }}$ in aikinite was calculated according procedure proposed by Makovicky, Makovicky (1978).

\section{Results and discussion}

Nuffieldite is relatively common mineral at the studied locality. It forms lead-gray acicular crystals reaching up to $1 \mathrm{~cm}$ in size, which are enclosed together with chalcopyrite, pyrite, chlorite and tourmaline in quartz-siderite gangue. Aggregates of nuffieldite crystals (Fig. 2) reaching up to $2.5 \mathrm{~cm}$ have also been rarely observed. They are homogenous in BSE (Fig. 3, 4) and crystals or aggregates of nuffieldite are frequently replaced by minor amounts of aikinite, galena and native bismuth (Fig. 5, 6).

The obtained experimental powder diffraction data set

Table 2 Unit-cell parameters of nuffieldite (for the orthorhombic space group Pbnm)

\begin{tabular}{llrrrr}
\hline & & \multicolumn{1}{c}{$a[\AA]$} & \multicolumn{1}{c}{$b[\AA]$} & \multicolumn{1}{c}{$c[\AA]$} & \multicolumn{1}{c}{$V\left[\AA^{3}\right]$} \\
\hline Slovinky & this paper & $14.5313(16)$ & $21.454(2)$ & $4.0500(6)$ & $1262.58(19)$ \\
Les Houches & Moëlo et al. (1997) & $14.4949(23)$ & $21.4195(49)$ & $4.0420(15)$ & $1254.93(95)$ \\
Maleevskoe & Mozgova et al. (1994) & 14.602 & 21.375 & 4.026 & 1256.6 \\
Alice Arm & Kohatsu, Wuensch (1973) & $14.387(7)$ & $21.011(15)$ & $4.046(6)$ & 1223.0 \\
\hline
\end{tabular}

Table 3 Representative WDS analyses of nuffieldite from Slovinky (wt.\%)

\begin{tabular}{lrrrrrrrrrrrrrrr}
\hline & 1 & 2 & 3 & 4 & 5 & 6 & 7 & 8 & 9 & 10 & 11 & 12 & 13 & 14 & 15 \\
\hline $\mathrm{Pb}$ & 36.16 & 37.02 & 35.67 & 36.44 & 35.53 & 35.85 & 36.59 & 36.54 & 36.37 & 35.60 & 34.99 & 35.60 & 35.90 & 36.35 & 36.50 \\
$\mathrm{Cu}$ & 6.20 & 6.29 & 6.13 & 6.40 & 6.09 & 6.38 & 6.28 & 6.30 & 6.25 & 6.26 & 6.07 & 5.95 & 6.23 & 6.31 & 6.29 \\
$\mathrm{Sb}$ & 3.76 & 4.03 & 3.48 & 3.59 & 3.19 & 3.80 & 4.10 & 4.09 & 3.77 & 3.72 & 3.10 & 3.26 & 3.64 & 3.47 & 4.16 \\
$\mathrm{Bi}$ & 36.10 & 35.50 & 37.73 & 36.25 & 37.40 & 36.04 & 35.92 & 35.61 & 36.12 & 36.55 & 37.07 & 36.92 & 36.82 & 36.66 & 35.55 \\
$\mathrm{~S}$ & 17.33 & 17.32 & 17.44 & 17.44 & 17.37 & 17.30 & 17.42 & 17.45 & 17.24 & 17.34 & 16.90 & 16.93 & 17.27 & 17.23 & 17.30 \\
$\mathrm{Se}$ & 0.14 & 0.18 & 0.20 & 0.00 & 0.19 & 0.26 & 0.10 & 0.13 & 0.00 & 0.10 & 0.57 & 0.55 & 0.23 & 0.15 & 0.11 \\
\hline total & 99.69 & 100.34 & 100.65 & 100.12 & 99.77 & 99.63 & 100.41 & 100.12 & 99.75 & 99.57 & 98.70 & 99.21 & 100.09 & 100.17 & 99.91 \\
\hline $\mathrm{Pb}$ & 2.308 & 2.341 & 2.258 & 2.321 & 2.276 & 2.297 & 2.311 & 2.318 & 2.314 & 2.277 & 2.272 & 2.289 & 2.284 & 2.312 & 2.315 \\
$\mathrm{Cu}$ & 1.290 & 1.297 & 1.265 & 1.329 & 1.272 & 1.333 & 1.293 & 1.303 & 1.296 & 1.306 & 1.285 & 1.248 & 1.292 & 1.309 & 1.301 \\
$\mathrm{Sb}$ & 0.408 & 0.434 & 0.375 & 0.389 & 0.348 & 0.414 & 0.441 & 0.442 & 0.408 & 0.405 & 0.342 & 0.357 & 0.394 & 0.376 & 0.449 \\
$\mathrm{Bi}$ & 2.284 & 2.226 & 2.368 & 2.290 & 2.376 & 2.289 & 2.249 & 2.240 & 2.278 & 2.318 & 2.386 & 2.354 & 2.322 & 2.312 & 2.236 \\
$\mathrm{~S}$ & 7.146 & 7.077 & 7.132 & 7.179 & 7.191 & 7.161 & 7.108 & 7.154 & 7.087 & 7.167 & 7.089 & 7.035 & 7.099 & 7.082 & 7.091 \\
$\mathrm{Se}$ & 0.023 & 0.030 & 0.033 & 0.000 & 0.032 & 0.044 & 0.017 & 0.022 & 0.000 & 0.017 & 0.097 & 0.093 & 0.038 & 0.025 & 0.018 \\
\hline calculated empirical formulas are based on $\mathrm{Pb}+\mathrm{Bi}+\mathrm{Sb}=5$ apfu & & & & & & & &
\end{tabular}

calculated empirical formulas are based on $\mathrm{Pb}+\mathrm{Bi}+\mathrm{Sb}=5$ apfu

Fig. 7 Variation of $\mathrm{Sb}$ vs. Bi (at.\%) in nuffieldite from Slovinky-Doštavná.

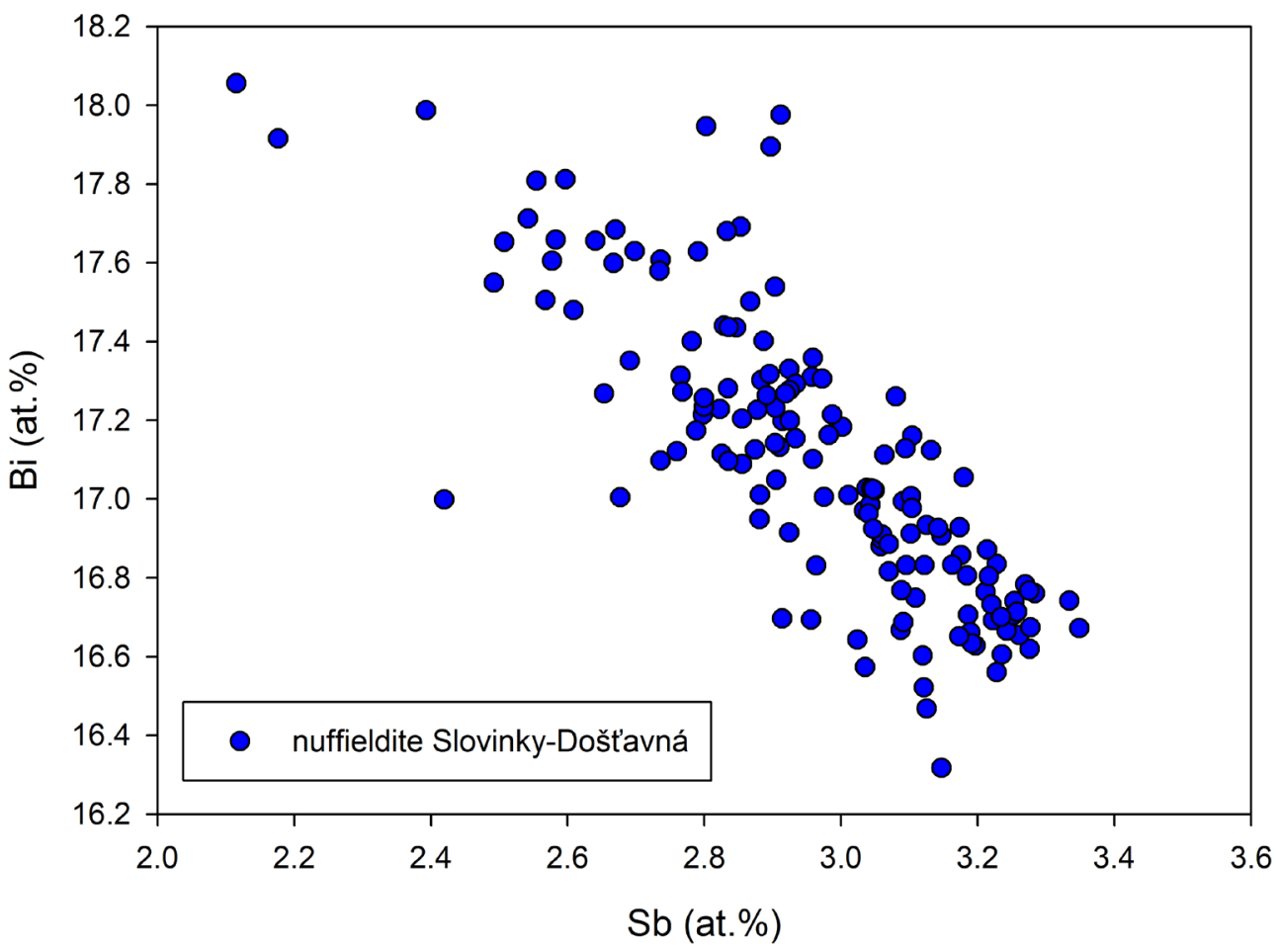




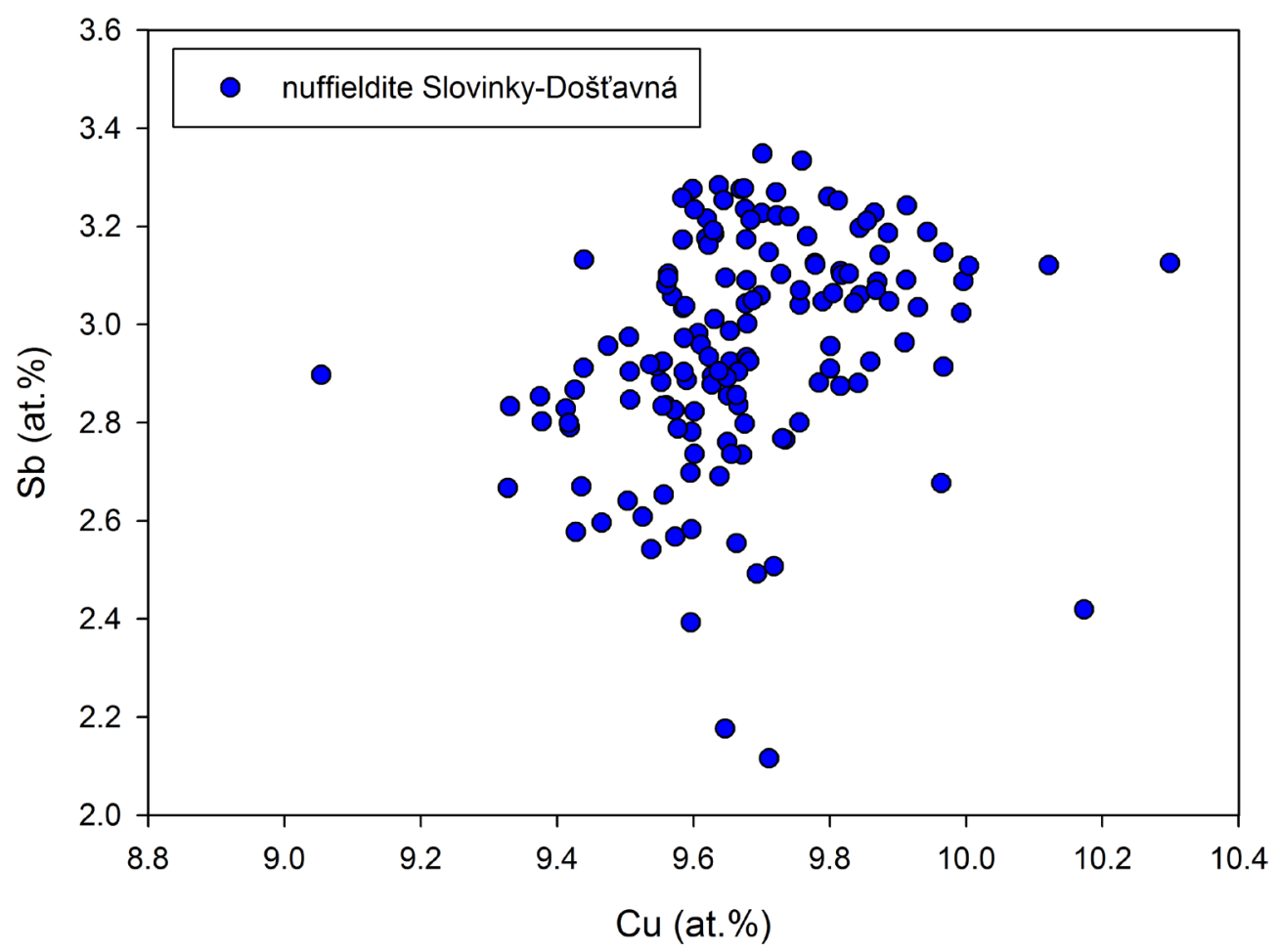

Fig. 8 Variation of $\mathrm{Cu}$ vs. Sb (at.\%) in nuffieldite from Slovinky-Doštavná.

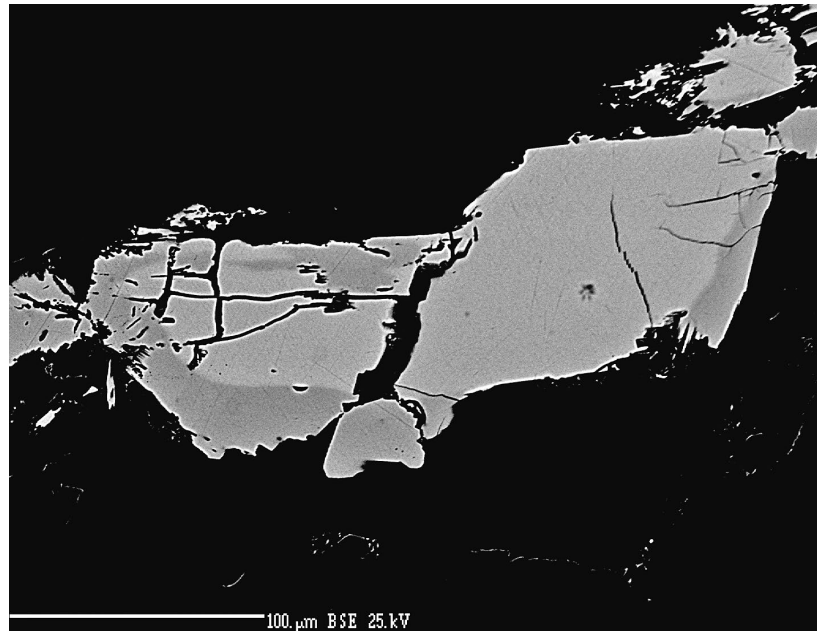

Fig. 9 Aikinite (dark gray) replaces nuffieldite (light gray) in quartz (black). BSE image by J. Sejkora.

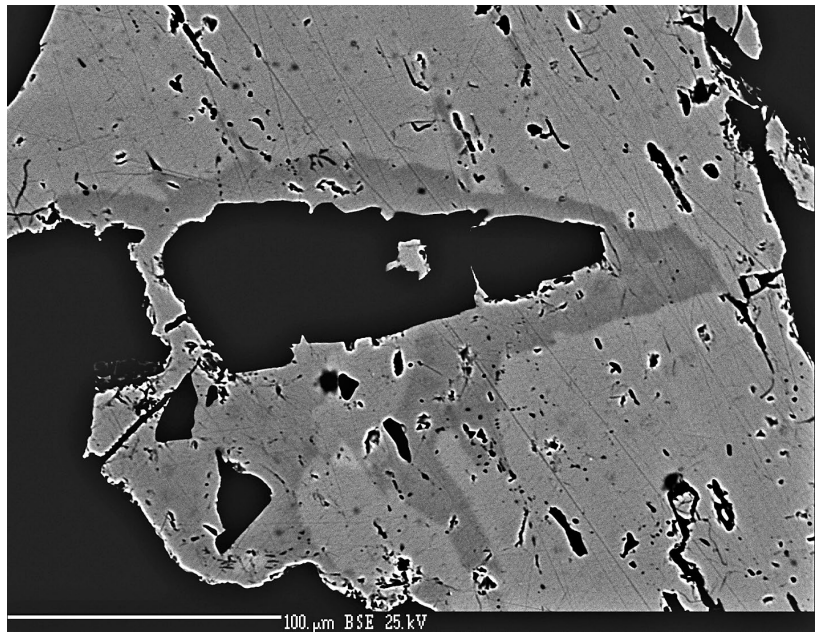

Fig. 10 Aikinite (dark gray) replaces nuffieldite (light gray) in quartz (black). BSE image by J. Sejkora.

Table 4 Representative WDS analyses of aikinite from Slovinky (wt.\%)

\begin{tabular}{|c|c|c|c|c|c|c|c|c|c|c|c|c|c|c|c|}
\hline & 1 & 2 & 3 & 4 & 5 & 6 & 7 & 8 & 9 & 10 & 11 & 12 & 13 & 14 & 15 \\
\hline $\mathrm{Pb}$ & 34.06 & 35.32 & 33.84 & 34.49 & 34.11 & 33.90 & 34.00 & 34.39 & 33.62 & 34.40 & 34.02 & 34.70 & 34.99 & 34.48 & 34.86 \\
\hline u & 10.61 & 10.37 & 10.49 & 10.49 & 10.26 & 10.29 & 10.21 & 10.44 & 10.00 & 10.53 & 10.44 & 9.56 & 9.95 & 10.45 & 10.33 \\
\hline$b$ & 46 & 0.56 & 0.57 & 0.25 & 18 & .25 & 0.32 & 0.23 & 0.56 & 0.54 & 0.38 & 1.05 & 1.39 & 0.47 & 0.63 \\
\hline $3 i$ & 37.06 & 37.19 & 37.20 & 36.63 & 37.88 & 37.89 & 37.94 & 36.84 & 39.01 & 36.89 & 37.50 & 36.76 & 35.88 & 37.48 & 37.45 \\
\hline & 17.25 & 17.29 & 17.42 & 16.87 & 17.17 & 17.19 & 17.09 & 17.00 & 17.70 & 17.15 & 17.34 & 17.29 & 17.22 & 17.20 & 17.42 \\
\hline $\mathrm{Se}$ & 0.09 & 0.14 & 0.00 & 0.24 & 0.11 & 0.08 & 0.14 & 0.09 & 0.00 & 0.00 & 0.10 & 0.12 & 0.10 & 0.00 & 0.00 \\
\hline otal & 99.53 & 100.87 & 99.52 & 98.97 & 99.71 & 99.60 & 99.70 & 98.99 & 100.89 & 99.51 & 99.78 & 99.48 & 99.53 & & 100.69 \\
\hline$b$ & 3.792 & 3.903 & 3.767 & 3.881 & 3.809 & 3.781 & 3.788 & 3.868 & 3.697 & 3.830 & 3.787 & 3.901 & 3.906 & 3.819 & $3.84 \varepsilon$ \\
\hline u & 3.852 & 3.737 & 3.807 & 3.849 & 3.736 & 3.743 & 3.709 & 3.828 & 3.586 & 3.822 & 3.790 & 3.504 & 3.622 & 3.774 & 3.718 \\
\hline b & 0.087 & 0.105 & 0.108 & 0.048 & 0.034 & 0.047 & 0.061 & 0.044 & 0.105 & 0.102 & 0.072 & 0.201 & 0.264 & 0.089 & 0.118 \\
\hline & 4.091 & 4.075 & 4.105 & 4.087 & 4.194 & 4.191 & 4.191 & 4.108 & 4.254 & 4.072 & 4.139 & 4.097 & 3.972 & 4.115 & 4.095 \\
\hline & 12.410 & 12.346 & 12.529 & 12.267 & & & & & 12.578 & & 12.474 & 12.558 & 12.423 & 12.308 & 12.42 \\
\hline $\mathrm{Se}$ & 0.026 & 0.041 & 0.000 & 0.071 & 0.032 & 0.023 & 0.041 & 0.027 & 0.000 & 0.000 & 0.029 & 0.035 & 0.029 & 0.000 & 0.000 \\
\hline aik & 96 & 95 & 95 & 97 & 94 & 94 & 94 & 96 & 91 & 96 & 95 & 93 & 94 & 95 & \\
\hline
\end{tabular}

calculated empirical formulas are based on $(\mathrm{Cu}+\mathrm{Pb}) / 2+(\mathrm{Sb}+\mathrm{Bi})=8$ apfu 
of nuffieldite from Slovinky given in Table 1 agrees well with the X-ray pattern calculated from the single-crystal data of nuffieldite from Les Houches in France (Moëlo et al. 1997); some observed differences in intensities of the individual diffraction maxima are caused probably by preferred orientation of the sample and small amount of sample available for experiment. The refined unit-cell parameters of studied nuffieldite are compared in Table 2 with published data.

The chemical composition of 11 different specimens of nuffieldite was quantitatively analysed and representative analyses as well as calculated empirical formulas are given in Table 3 (all 145 analyses are available in supplementary file). Moëlo (1989); Maurel, Moëlo (1990) and Moëlo et al. (1997) pointed that Sb content is essential for stability of nuffieldite in nature and excess of $\mathrm{Cu}$ is typical. Based on this they redefined empirical formula of nuffieldite as $\mathrm{Cu}_{1+x-}$ $\mathrm{Pb}_{2} \mathrm{Bi}_{2}\left(\mathrm{~Pb}_{x} \mathrm{Sb}_{y} \mathrm{Bi}_{1-x-y}\right) \mathrm{S}_{7}$, with $x$ close to 0.37 and $y$ ranging between 0.19 and 0.55 . Aikinite-type $\mathrm{Cu}+\mathrm{Pb}=(\mathrm{Bi}, \mathrm{Sb})+\mathrm{va}$ cancy substitution is characteristic for nuffieldite (Maurel, Moëlo 1990; Pršek et al. 2006; Pršek 2008). The $x$ value in studied samples from Slovinky-Došt'avná varies between 0.18 to 0.44 (with average of 0.30 ) and $y$ value is ranging from 0.28 to 0.45 (with average of 0.40 ), which is in line with published data on chemical composition of nuffieldite (e.g. Moëlo 1989; Harris 1993; Mozgova et al. 1994; Moëlo et al. 1997; Pršek et al. 2006; Izumino et al. 2014). There is obvious negative correlation between $\mathrm{Sb}$ vs. Bi contents (Fig. 7), but contents of Cu vs. Sb has clearly positive trend (Fig. 8). Minor amounts of Se (reaching up to 0.10 apfu) were also detected. The average ( $n=145$ analyses) empirical formula of nuffieldite from Slovinky-Doštavná based on $\mathrm{Pb}+\mathrm{Bi}+\mathrm{Sb}=5$ apfu is corresponding to $\mathrm{Cu}_{1.30} \mathrm{~Pb}_{2.00}$ $\mathrm{Bi}_{2.00}\left(\mathrm{~Pb}_{0.30} \mathrm{Bi}_{0.30} \mathrm{Sb}_{0.40}\right)_{1.00}\left(\mathrm{~S}_{7.12} \mathrm{Se}_{0.03}\right)_{7.15}$.

Aikinite forms microscopic, anhedral to subhedral grains or aggregates reaching up to $300 \mu \mathrm{m}$ in size (Fig. 6, 9, 10 ), which are replacing nuffieldite. It is mostly associated with chalcopyrite, galena and native bismuth. Representative WDS analyses of aikinite from Slovinky are shown in Table 4 (all 29 analyses are available in supplementary file). Besides of dominant contents of $\mathrm{Cu}, \mathrm{Pb}, \mathrm{Bi}$ and $\mathrm{S}$ minor amounts of Sb (up to $0.26 \mathrm{apfu}$ ) and also Se (up to $0.07 \mathrm{apfu}$ ) were observed. The calculated hypothetical percentage of the aikinite end member $n_{\text {aik }}$ is ranging from 91 to 97 (with average of 95). The average $(n=29)$ empirical formula of studied aikinite based on $(\mathrm{Cu}+\mathrm{Pb}) / 2+(\mathrm{Sb}+$ $\mathrm{Bi})=8$ apfu is $\mathrm{Pb}_{3.83} \mathrm{Cu}_{3.76}\left(\mathrm{Bi}_{4.12} \mathrm{Sb}_{0.09}\right)_{4.21}\left(\mathrm{~S}_{12.40} \mathrm{Se}_{0.03}\right)_{12.03}$.

\section{Conclusions}

A new occurrence of rare $\mathrm{Cu}, \mathrm{Pb}$ sulphosalt, nuffieldite was identified at the siderite-type hydrothermal carbonate-quartz vein in Došt'avná near Slovinky. It is associated with chalcopyrite, pyrite, aikinite, galena, native bismuth, tourmaline and chlorite.

\section{Acknowledgements}

The authors wish to thank to Pavel Škácha for the microphotography of studied samples and to Zdeněk Dolníček for his kind help with the analytical work. This study was financially supported by the Ministry of Culture of the Czech Republic (long-term project DKRVO 2019-2023/1. II.c; National Museum, 00023272) and by VEGA project (2/0028/20)

\section{References}

AnTAL B (1991) Bi-sulfosoli ložiska Slovinky. Miner Slov 23(4): 368

AntAl B (2002a) Tennantite from the vein Mayer, baňa Mária deposit in Rožňava, Spišsko-gemerské rudohorie Mts. Slovak Geol Mag 8(3-4): 195-204

ANTAL B (2002b) Trends in chemical composition of tetrahedrite from the deposit Jedlovec (Fichtenhübel), Spišsko-gemerské rudohorie Mts. Slovak Geol Mag 8(3-4): 205-217

Bajaník Š, Ivanička J, Mello J, Pristaš J, Reichwalder P, Snopko L, VozÁr J, VozÁrová A (1984) Geologická mapa Slovenského rudohoria, východná čast' 1:50 000. ŠGÚDŠ, Bratislava

BEŇKA J, SIMAN P (1994) Identifikácia nových minerálov Cu, $\mathrm{Pb}, \mathrm{Bi}, \mathrm{Sb}$ v žilách Brdárky. Geol Práce Spr 99: 23-37

BERNARD JH (1958) Chemismus a velikost mřižkových konstant spišsko-gemerských tetraedritů. Rozpr Čs Akad Věd R mat prír Věd 68: 1-74

BeRnARD JH (1961) Mineralogie und Geochemie der SideritSchwerspatgänge mit Sulfiden im Gebiet von Rudňany (Tschechoslowakei). Geol Práce Zoš 58: 1-222

Bernard JH (1964) Cosalit $\left(\mathrm{Pb}_{2} \mathrm{Bi}_{2} \mathrm{~S}_{5}\right)$ ze sideritové žily v Prakovcích ve Spišsko-gemerském rudohoří. Geol Práce Zpr 33: 43-52

Burnham CHW (1962) Lattice constant refinement. Carnegie Inst Washington Year Book 61: 132-135

Cambel B, Jarkovský J, Faith L, Forgáč J, Hovorka D, Hrnčárová M, Hurný J, Ivan P, Karoli A, Král J, Litavec J, Matula I, Mihalov J, Popreñák J, Rényı K, Rojkovič I, RozloŽNiK L, SASVÁRI T, SAVČENKOVÁ L, SPIŠIAK J, ŠMEJKAL V, Turan J, Turanová L, Varček C, Žabka M, Žukov F (1985) Rudnianské rudné pole. Veda, Bratislava, 1-363

Chovan M, Háber M, Jeleñ S, Rojkovič I (1994) Ore textures in the Western Carpathians. Slovac Academic Press, Bratislava, 1-219

Grecula P, Abony A, Abonylová M, Antaš J, Bartalský B, BartalskÝ J, DianIŠKa I, ĎuĎa R, GaRgulák M, GaZdaČKo L', HUdÁČEK J, KoBULSKÝ J, LöRINCZ L, MACKO J, NÁVESŇÁK D, Németh Z, Novotný L, Radvanec M, Rojkovič I, RozLOŽNIK L, VARČEK C, ZLOCHA Z (1995) LoŽiská nerastných surovín Slovenského rudohoria. Zväzok 1. Geocomplex, Bratislava, 1-834

HÁBER M (1980) Mineralogische-geochemische und paragenetische Erforschung hydrothermaler Gänge im Gebiet zwischen Prakovce und Kojšov (SGR). Záp Karpaty, Sér Miner Petrogr Geoch Metalog 7: 7-131

HÁBER M (1978) Mineralogicko-paragenetické pomery na ložisku Novoveská Huta Cu v Spišsko-gemerskom rudohorí. In: Hornická Příbram ve vědě a technice, sek geologie: 411-423

HÁBER M, StREŠKo V (1969) Ein neues Kobellit-Vorkommen in dem Zips-Gömörer Erzgebirge. Geol Zbor Geol Carpath 20(1): 133-151

HÁber M, KRIŠTín J, RoJkovič I (1993) The tennantite-tetrahedrite series in Permian formations of the Western Carpathians. Geol Carpath 45(1): 11-28

HaK J, KUPKA F (1958) Identifikace kobellitu z Hummelu ve Spišsko-gemerském Rudohoří. Čas Mineral Geol 3(1): $16-20$

HARRIS DC (1993) Confirmation of antimony in co-type nuffieldite, Lime Creek, British Columbia, and a second Canadian occurrence at Izok Lake, Northwest Territories. Geol Surv Can pap 93-1E: 9-10

HuRNÝ J (1973) Štúdium mineralógie, paragenézy a geochémie na hydrotermálnych rudných žilách južne od Rudnian. Rigorózna práca, archív ŠGÚDŠ-Geofond, Bratislava, 1-107 (38783) 
HURNý J (1974) Príspevok k štúdiu mineralogicko-paragenetických pomerov hydrotermálnych rudných žíl južne od Rudnian. Miner Slov 6(2): 113-125

HuRnÝ J, KRIŠTín J (1978) Aikinite and xenotime from Slovinky (Spišsko-gemerské rudohorie). Geol Zbor Geol Carpath 29(1): 167-171

IZUMINO Y, NAKASHIMA K, NAGASHIMA M (2014) Cuprobismutite group minerals (cuprobismutite, hodrušite, kupčíkite and paděraite), other Bi-sulfosalts and Bi-tellurides from the Obari mine, Yamagata Prefecture, Japan. J Mineral Petrol Sci 109(4): 177-190

JeLEŇ M (1991) Chemické zloženie sulfosolí zo žily Strieborná v Rožňave. Miner Slov 23(4): 369

KoHATSU I, WUENSCH BJ (1973) The crystal structure of nuffieldite, $\mathrm{Pb}_{2} \mathrm{Cu}(\mathrm{Pb}, \mathrm{Bi}) \mathrm{Bi}_{2} \mathrm{~S}_{7}$. Zeit Kristallogr 138: 343365

KozuB G, BudzyŃ B, PršEK J (2011) Hydrothermal copperbismuth mineralization from the Joremeny adit, Dobšiná, Slovak Republic-preliminary report. Mineralogia, Spec Pap 38: 123-124

KupČík V, SCHneIder A, VARČEK C (1969) Chemismus von einigen Bi-Sulfosalzen aus dem Zips-Gömörer Erzgebirge. N Jb Miner, Mh 10: 445-454

MAKOVICKY E, MAKOVICKY M (1978) Representation of compositions in the bismuthinite-aikinite series. Can Mineral 16(3): 405-409

Maurel C, MoËLo Y (1990) Synthèse de la nuffieldite dans le système Bi-Pb-Sb-Cu-S. Can Mineral 28(4): 745749

Mikuš T, Kondela J, Jacko S, Milovská S (2018) Garavellite and associated sulphosalts from the Strieborná vein in the Rožňava ore field (Western Carpathians). Geol Carpath 69(3): 221-236

MıKUŠ T, BAKOS F, HöNIG S (2019) Bismuth sulphosalts from the siderite-sulphidic and As-Co mineralization in Medzev area, Slovakia. Acta Geol Slov 11(2): 91-102

Mıškovıc J (1990) Mineralogicko-paragenetické pomery na ortut'ovom ložisku Rákoš. Miner Slov 22(1): 68

Mıškovıc J (1991) Tetraedrity z dobšinského rudného pol'a. Miner Slov 23(4): 367-368

MoËLO Y (1989) Antimoine dans la nuffieldite associée á de la friedrichite (commune des Houches, Alpes de Haute-Savoie); redéfinition cristallochimique de la nuffieldite. C R Acad Sci Paris 309(2): 1659-1664

Moëlo Y, Meerschaut A, Makovicky E (1997) Refinement of the crystal structure of nuffieldite, $\mathrm{Pb}_{2} \mathrm{Cu}_{1.4}\left(\mathrm{~Pb}_{0.4}\right.$ $\left.\mathrm{Bi}_{0.4} \mathrm{Sb}_{0.2}\right) \mathrm{Bi}_{2} \mathrm{~S}_{7}$ : Structural relationships and genesis of complex lead sulfosalt structures. Can Mineral 35(6): 1497-1508

Mozgova NN, Nenasheva SN, Borodaev YS, Yudovskaya MA (1994) Nuffieldite from the Maleevskoe massive sulfide dposit, Russia. Can Mineral 33(2): 359-364

MuMmE W, ŽÁK L (1983) Second occurrence of krupkaite in Czechoslovakia. Čas Mineral Geol 28(1): 61-63

NovÁk F (1959) Tetraedrit z ložiska Mária u Rožňavy. Geol Práce Zoš 56: 217-246

Novák F (1961) Kobellit ze žíly Mária u Rožňavy. Věst Ústř Úst geol 36(2): 97-107

NovÁK F (1962) Výskyt jamesonitu a bournonitu na sideritových žilách u Rožňavy. Čas Mineral Geol 7(3): 373-281

NovÁK F (1967) Chemismus tetraedritu ze VII. a X. patra žíly Mária u Rožňavy. Čas Mineral Geol 12(1): 49-57

ONDRUŠ P (1993) ZDS - A computer program for analysis of X-ray powder diffraction patterns. Materials Science Forum, 133-136, 297-300, EPDIC-2. Enchede

PADĚRA K, BouŠKA V, PELIKÁN J (1955) Rezbanyit aus Dobšiná in der Ostslowakei, ČSR. Chem Erde 17(4): 329-340
Peterec D (1990) Výskyt rumelky a Hg-tennantitu pri Vyšnom Klátove. Miner Slov 22(2): 189-190

Pouchou JL, PICHOIR F (1985) "PAP" ( $\varphi \rho Z)$ procedure for improved quantitative microanalysis. In: Microbeam Analysis (J. T. Armstrong, ed.). San Francisco Press, San Francisco, 104-106

PRŠEK J (2008) Chemické zloženie a kryštalochémia Bi sulfosolí z hydrotermálnych mineralizácií kryštalinika Západných Karpát. Univerzita Komenského, Bratislava, 1-108

PRŠEK J, BIROŇ A (2007) Jaskólskiit a sprievodné sulfosoli zo žily Aurélia-Rožňava, Spišsko-gemerské rudohorie. Miner Slov 39(2): 141-146

PRŠEK J, LAUKO L' (2009) Hydrotermálna žilná mineralizácia v Zlatej Idke. Miner Slov 41(2): 133-150

Pršek J, Peterec D (2008) Bi-Se-Te mineralization from Úhorná (Spišsko Gemerské Rudohorie Mts., Slovakia): A preliminary report. Mineralogia 39(3-4): 87-103

PrŠEK J, MAKovicky M, Chovan M, SMIRnov A (2006) A note on chemical composition of nuffieldite solid-solution from sulphide mineralizations (Western Carpathians, Slovakia). Mineral Polon 37(1): 51-60

RoJkovıč I (1997) Uranium Mineralization in Slovakia. Acta geol Univ Comen, Monogr Ser 1-117

Sejkora J, Ozdín D, Laufek F, Plášil J, Litochleb J (2011) Marrucciite, a rare Hg-sulfosalt from the Gelnica ore deposit (Slovak Republic), and its comparison with the type occurrence at Buca della Vena mine (Italy). J Geosci 56(4): 399-408

ŠteVko M, SEJKora J (2020) Sb-enriched association of $\mathrm{Ni}$ arsenides and sulfarsenides from the Zemberg-Terézia vein system near Dobšiná (Western Carpathians, Slovak Republic). Bull Mineral Petrolog 28(1): 105-115

ŠteVko M, SeJkora J, Peterec D (2015) Grumiplucite from the Rudnany deposit, Slovakia: a second world-occurrence and new data. J Geosci 60(4): 269-281

TRDLIČKA Z (1967) Mineralogické studium tetraedritu Z ložiska Fichtenhübel (Spišsko-gemerské rudohoří). Čas Mineral Geol 12(2): 115-121

TRDLIČKA Z, KUPKA F (1959) Kobellit a ryzí vizmut z lokality Fichtenhübel na Slovensku. Sbor Ústř Úst geol (Praha) $453-464$

TRDLIČKA Z, KVAČEK M, KUPKA F (1962) Mineralogicko-chemický výzkum kobellitu ze sideritových žil rudní oblasti Fichtenhübel (Spišsko-gemerské rudohoří). Čas Mineral Geol 7(4): 432-433

VÁCLAV J (1964) Identifikácia kobellitu zo žily Dávid pri Henclovej. Geol Práce Zpr 31: 91-94

VARČEK C (1957) Prehl'ad paragenetických pomerov rudných ložísk Gemera. Geol Práce Zoš 46: 107-131

VARČEK C (1959) Paragenetické pomery žily Mária v Rožňave. Geol Práce Zoš 55: 181-213

VARČEK C (1960) Paragenetické pomery žily Bernardi pri Rožňave. Acta Geol Geogr Univ Comen, Geol 4: 107155

VARČEK C (1962) Vývoj hydrotermálnej mineralizácie Spišsko-gemerského rudohoria v čase a priestore. Geol Práce Zoš 61: 101-112

Yvon K, Jeitschko W, Parthé E (1977) Lazy Pulverix, a computer program for calculation X-ray and neutron diffraction powder patterns. J Appl Cryst 10: 73-74

ZÁBRANSKÝ F, RADZO V (1966) Výskyt kobellitu a paragenetické pomery na žile Oriešková, severne od Nižného Medzeva. Sbor Východoslov Múz (Košice) Sér A, 7: 25-37

ZIMÁNYI K (1914) Arsenopyrit und Bournonit von Rozsnyó. Z Kristallogr Mineral 54(4): 578-583 